\title{
Erratum to: Orienting attention in visual space by nociceptive stimuli: investigation with a temporal order judgment task based on the adaptive PSI method
}

\author{
Lieve Filbrich $^{1} \cdot$ Andrea Alamia $^{1} \cdot$ Soline Burns $^{1} \cdot$ Valéry Legrain $^{1}$
}

Published online: 4 July 2017

(C) Springer-Verlag GmbH Germany 2017

\section{Erratum to: Exp Brain Res (2017) 235:2069-2079 DOI 10.1007/s00221-017-4951-2}

In the Abstract, the second sentence should read "This study investigated if, and in which time window, a nociceptive stimulus can attract attention to its location on the body, independently of voluntary control, to facilitate the processing of visual stimuli occurring in the same side of space as the limb on which the nociceptive stimulus was applied" (instead of "This study investigated if, and in which time window, a nociceptive stimulus can attract attention to its location on the body, independently of voluntary control, to facilitate the processing of visual stimuli occurring in the same side of space as the limb on which the visual stimulus was applied").
The online version of the original article can be found under doi:10.1007/s00221-017-4951-2.

Lieve Filbrich

lieve.filbrich@uclouvain.be

Institute of Neuroscience, Université catholique de Louvain, Brussels, Avenue Mounier 53, boite COSY B1.53.04,

1200 Brussels, Belgium
In the Methods/Measures section, the sentence "Too estimate the logistic function we used the PSI method (Kontsevich and Tyler 1999), [...] at each trial" should read "To estimate the logistic function we used the PSI method (Kontsevich and Tyler 1999), [...] at each trial".

In the Results/PSS section, readers should refer to "Fig. 1A" instead of Fig. 1a for an illustration of the simple $t$ tests on PSS values.

In the Results/Slope section, degrees of freedom for the non-significant main effect of the factor time interval should be $F(1, \underline{28})$ instead of $F(1,18)$. 